JOURNAL OF THE AMERICAN MATHEMATICAL SOCIETY

Volume 20, Number 4, October 2007, Pages 989-1001

S 0894-0347(07)00565-6

Article electronically published on April 2, 2007

\title{
AN INFINITE DIMENSIONAL AFFINE NIL ALGEBRA WITH FINITE GELFAND-KIRILLOV DIMENSION
}

\author{
T. H. LENAGAN AND AGATA SMOKTUNOWICZ
}

\section{INTRODUCTION}

In their famous paper 2], Golod and Shafarevich gave a construction which yields, over arbitrary fields, infinite dimensional, finitely generated nil algebras that are not nilpotent algebras. In fact, the algebras arising from the Golod-Shafarevich construction have exponential growth. In view of this, Lance Small has asked whether there is a finitely generated, infinite dimensional nil algebra with finite Gelfand-Kirillov dimension, which is not nilpotent, 4]. (This question has also been asked by other people; see, for example [1, page 87].) Small's question is answered in this paper: we construct, over an arbitrary countable field, a finitely generated, non-nilpotent, nil algebra with Gelfand-Kirillov dimension not exceeding 20.

In what follows $K$ is a countable field and $A$ is the free $K$-algebra in three noncommuting indeterminates $x, y$ and $z$. The set of monomials in $x, y, z$ is denoted by $M$, and $M(n)$ denotes the set of monomials of degree $n$, for each $n \geq 0$. Thus, $M(0)=\{1\}$, and for $n \geq 1$ the elements in $M(n)$ are of the form $x_{1} \ldots x_{n}$, where $x_{i} \in\{x, y, z\}$. The $K$-subspace of $A$ spanned by $M(n)$ will be denoted by $H(n)$, and elements of $H(n)$ will be called homogenous polynomials of degree $n$. Every polynomial $f \in A$ such that $\operatorname{deg}(f)=d$ can be uniquely presented in the form $f=f_{0}+f_{1}+\ldots+f_{d}$, where $f_{i} \in H(i)$. The elements $f_{i}$ are the homogeneous components of $f$, and $\operatorname{deg}(f)$ denotes the degree of the polynomial $f$. A right ideal $I$ of $A$ is homogeneous if for every $f \in I$ all homogeneous components of $f$ are in $I$. Let $V$ be a linear space over $K$; then $\operatorname{dim}_{K} V$ denotes the dimension of $V$ over $K$. The Gelfand-Kirillov dimension of an algebra $R$ is denoted by $\operatorname{GKdim}(R)$. For elementary properties of Gelfand-Kirillov dimension we refer to [3].

\section{EnUmerating ELEMENTS}

Let $\bar{A}$ be the subalgebra of $A$ consisting of polynomials with constant term equal to zero. As usual, $\mathbb{N}$ denotes the set of natural numbers.

The aim is to present an algebra with the desired properties as $\bar{A} / E$ for a suitable ideal $E$. The ideas develop from ideas in earlier papers by the second author, but

Received by the editors May 25, 2005.

2000 Mathematics Subject Classification. Primary 16Nxx, 16P90.

Key words and phrases. Nil algebra, growth of algebras, Gelfand-Kirillov dimension.

The first author acknowledges support by Leverhulme Grant F/00158/X.

Part of this work was done while the second author was visiting the University of Edinburgh, with support from the Edinburgh Mathematical Society. The second author acknowledges support by an EPSRC Advanced Fellowship EP/D071674/1.

(C)2007 American Mathematical Society 989

Reverts to public domain 28 years from publication 
we have to define several sets of subspaces carefully in order to be able to control the growth of the algebra we have in mind.

We start with two results derived from similar results in [6].

Lemma 1. Let $K$ be a countable field, and let $\bar{A}$ be as above. Then there exists a set $Z \subseteq \mathbb{N}$, with all $i \in Z$ being greater than or equal to 5 , such that the elements of $\bar{A}$ can be enumerated as $f_{i}$ for $i \in Z$ (that $i s, \bar{A}=\left\{f_{i}\right\}_{i \in Z}$ ) and such that $2^{2^{i}}>3^{6 t_{i}}$ for each $i \in Z$, where $t_{i}$ is the degree of $f_{i}$.

Proof. The field $K$ is countable and the algebra $A$ is finitely generated over $K$, so the elements of $\bar{A}$ can be enumerated: say $\bar{A}=\left\{g_{1}, g_{2}, \ldots\right\}$. We now define an increasing function $\theta: \mathbb{N} \longrightarrow \mathbb{N}$ as follows. Set $\theta(1):=\min \{i \in \mathbb{N} \mid i>$ $\left.4,2^{2^{i}}>3^{6 \operatorname{deg}\left(g_{1}\right)}\right\}$. Suppose that we have defined $\theta:\{1, \ldots, n\} \longrightarrow \mathbb{N}$ such that $2^{2^{\theta(i)}}>3^{6 \operatorname{deg}\left(g_{i}\right)}$, for each $i=1, \ldots, n$. Then set $\theta(n+1):=\min \{s \mid s>$ $\theta(1), \ldots, \theta(n)$ and $\left.2^{2^{s}}>3^{6 \operatorname{deg}\left(g_{n+1}\right)}\right\}$. If we now rename the elements of $\bar{A}$ by setting $f_{\theta(s)}:=g_{s}$, then we have a listing of the elements of $\bar{A}$ with the required properties.

Given a subset $S \subseteq H(n)$, for some $n$, let $B_{n}(S)$ denote the right ideal of $A$ generated by the set $\bigcup_{k=0}^{\infty} M(n k) S$; that is,

$$
B_{n}(S)=\sum_{k=0}^{\infty} M(n k) S A .
$$

Theorem 2. Let $Z,\left\{f_{i}\right\}_{i \in Z}$ be as in Lemma 1. Let $i \in Z$, and let $I$ be the twosided ideal generated by $f_{i}{ }^{10 w_{i}}$ where $w_{i}=4.2^{2^{i}}$. Then there is a linear $K$-space $F_{i} \subseteq H\left(2^{2^{i}}\right)$ such that $I \subseteq B_{w_{i}}\left(F_{i}\right)$ and $\operatorname{dim}_{K}\left(F_{i}\right)<2^{2^{i+1}}-2$.

Proof. Apply [6, Theorem 2] with $f=f_{i}, r=2^{2^{i}}, w=w_{i}=4.2^{2^{i}}$, and put $F_{i}=\operatorname{span}_{K} F$. Note that these choices of $f, r, w$ satisfy the hypotheses of 6 , Theorem 2], by Lemma 1.

\section{Definition of $U\left(2^{n}\right)$ And $V\left(2^{n}\right)$}

Set $S:=\left\{\left[2^{i}-i-1,2^{i}-1\right] \mid i=5,6,7, \ldots\right\}$.

Theorem 3. Let $Z, F_{i}$ be as in Theorem 2. Then there are K-linear subspaces $U\left(2^{n}\right)$ and $V\left(2^{n}\right)$ of $H\left(2^{n}\right)$ such that for all $n>0$ we have:

1. $\operatorname{dim}_{K} V\left(2^{n}\right)=2$ if $n \notin S$.

2. $\operatorname{dim}_{K} V\left(2^{2^{i}-i-1+j}\right)=2^{2^{j}}$, for all $i>4$ and all $0 \leq j \leq i$.

3. $V\left(2^{n}\right)$ is generated by monomials.

4. $F_{i} \subseteq U\left(2^{2^{i}}\right)$ for every $i \in Z$.

5. $V\left(2^{n}\right)+U\left(2^{n}\right)=H\left(2^{n}\right)$ and $V\left(2^{n}\right) \cap U\left(2^{n}\right)=0$.

6. $H\left(2^{n}\right) U\left(2^{n}\right)+U\left(2^{n}\right) H\left(2^{n}\right) \subseteq U\left(2^{n+1}\right)$.

7. $V\left(2^{n+1}\right) \subseteq V\left(2^{n}\right) V\left(2^{n}\right)$.

Proof. We construct the sets $U\left(2^{n}\right)$ and $V\left(2^{n}\right)$ inductively. Set $V\left(2^{0}\right):=V(1)=$ $K x+K y$ and $U\left(2^{0}\right)=U(1):=K z$. Assume that we have defined $V\left(2^{m}\right)$ and $U\left(2^{m}\right)$ for $m \leq n$ in such a way that conditions 1-5 hold for all $m \leq n$ and conditions 6 and 7 hold for all $m<n$. Then we define $V\left(2^{n+1}\right)$ and $U\left(2^{n+1}\right)$ in the following way. Observe first that since $U(n) \cap V(n)=0$, then

$$
\{U(n) U(n)+U(n) V(n)+V(n) U(n)\} \cap\{V(n) V(n)\}=0 .
$$


Our next step is to make the following observation. If $\bar{V}, P \subseteq V(n) V(n)$ and $\bar{V} \cap P=0$, then

$$
\{U(n) U(n)+U(n) V(n)+V(n) U(n)+\bar{V}\} \cap P=0 .
$$

For, suppose that $c=c_{1}+c_{2} \in P$ with $c_{1} \in U(n) U(n)+U(n) V(n)+V(n) U(n)$ and $c_{2} \in \bar{V}$. We claim that $c=0$. Notice that $c \in P$ and $c_{2} \in \bar{V}$ implies that $c_{1}=c-c_{2} \in P+\bar{V} \subseteq V(n) V(n)$. On the other hand, $c-c_{2}=c_{1} \in$ $U(n) U(n)+U(n) V(n)+V(n) U(n)$. By the above observation, we get $c_{1}=0$ so that $c=c_{2} \in \bar{V}$. However, $c \in P$, so that $c \in P \cap \bar{V}=0$, as required.

Now we will define $V\left(2^{n+1}\right), U\left(2^{n+1}\right)$ inductively, in the following way. Consider the three cases:

1. $n \in S$ and $n+1 \in S$.

2. $n \notin S$.

3. $n \in S$ and $n+1 \notin S$.

Case 1. Suppose that $n \in S$ and $n+1 \in S$. Then we define $V\left(2^{n+1}\right):=$ $V\left(2^{n}\right) V\left(2^{n}\right)$; so condition 7 certainly holds. Notice that $V\left(2^{n+1}\right)$ is spanned by monomials, since $V\left(2^{n}\right)$ is spanned by monomials; so condition 3 holds. Moreover $\operatorname{dim}_{K} V\left(2^{n}\right)=\left(\operatorname{dim}_{K} V\left(2^{n}\right)\right)^{2}$. Since $n, n+1 \in S$, it follows that $n=2^{i}-i-1+j$ for some $i$ and some $0 \leq j<i$. By the inductive hypothesis, $\operatorname{dim}_{K} V\left(2^{n}\right)=2^{2^{j}}$. Now $\operatorname{dim}_{K} V\left(2^{n+1}\right)=\left(2^{2^{j}}\right)^{2}=2^{2^{j+1}}$, as required for condition 2 (condition 1 does not apply in this case). Set $U\left(2^{n+1}\right):=U\left(2^{n}\right) H\left(2^{n}\right)+H\left(2^{n}\right) U\left(2^{n}\right)$; so condition 6 certainly holds. It is now easy to check that condition 5 holds. Finally, observe that since $n+1 \in S$, we have $2^{n+1} \neq 2^{2^{i}}$ for every $i$, hence condition 4 is empty in this case and so holds trivially.

Case 2. Suppose that $n \notin S$. Then $\operatorname{dim}_{K} V\left(2^{n}\right)=2$, by the inductive hypothesis. Let $m_{1}, m_{2}$ be distinct monomials from $V\left(2^{n}\right) V\left(2^{n}\right)$. Set $V\left(2^{n+1}\right):=$ $K m_{1}+K m_{2}$. Then $\operatorname{dim}_{K} V\left(2^{n+1}\right)=2$, as required. Let $\bar{V} \subseteq V\left(2^{n}\right) V\left(2^{n}\right)$ be such that $\bar{V} \cap V\left(2^{n+1}\right)=0$ and $\bar{V}+V\left(2^{n+1}\right)=V\left(2^{n}\right) V\left(2^{n}\right)$. Set $U\left(2^{n+1}\right):=$ $U\left(2^{n}\right) V\left(2^{n}\right)+V\left(2^{n}\right) U\left(2^{n}\right)+U\left(2^{n}\right) U\left(2^{n}\right)+\bar{V}$. We see that $U\left(2^{n+1}\right) \cap V\left(2^{n+1}\right)=0$ and $U\left(2^{n+1}\right)+V\left(2^{n+1}\right)=H\left(2^{n+1}\right)$. Observe that since $n \notin S$, we get $2^{n+1} \neq 2^{2^{i}}$ for every $i$, and again 4 holds trivially.

Case 3. Suppose that $n \in S$ while $n+1 \notin S$. Then $n=2^{i}-1$ for some $i>1$. By the inductive hypothesis $\operatorname{dim}_{K} V\left(2^{n}\right)=\operatorname{dim}_{K} V\left(2^{2^{i}-1}\right)=\operatorname{dim}_{K} V\left(2^{2^{i}-i-1+i}\right)=$ $2^{2^{i}}$. Now $\operatorname{dim}_{K} V\left(2^{n}\right) V\left(2^{n}\right)=2^{2^{i+1}}$.

Assume first that $i \in Z$. We know that $F_{i}$ has a basis $\left\{f_{1}, \ldots, f_{s}\right\}$ for some $f_{1}, \ldots, f_{s} \in H\left(2^{2^{i}}\right)$ and $s<2^{2^{i+1}}-2$. Write each $f_{j}$ as $f_{j}=\bar{f}_{j}+g_{j}$ where $\bar{f}_{j} \in V\left(2^{n}\right) V\left(2^{n}\right)$ and $g_{j} \in V\left(2^{n}\right) U\left(2^{n}\right)+U\left(2^{n}\right) U\left(2^{n}\right)+U\left(2^{n}\right) V\left(2^{n}\right)$. Since $V\left(2^{n}\right) \cap U\left(2^{n}\right)=0$ this decomposition is unique. Let $P$ be a $K$-linear subspace of $V\left(2^{n}\right) V\left(2^{n}\right)$ such that $\bar{f}_{j} \in P$ for all $1 \leq j \leq s$ and $\operatorname{dim}_{K} P=2^{2^{i+1}}-2$.

Since $V\left(2^{n}\right) V\left(2^{n}\right)$ is spanned by monomials and $\operatorname{dim}_{K}\left(V\left(2^{n}\right) V\left(2^{n}\right)\right)=2^{2^{i+1}}$ while $\operatorname{dim}_{K} P=2^{2^{i+1}}-2$, then there are monomials $m_{1}, m_{2} \in V\left(2^{n}\right) V\left(2^{n}\right)$ such that $K m_{1}+K m_{2}+P=V\left(2^{n}\right) V\left(2^{n}\right)$ and $P \cap\left(K m_{1}+K m_{2}\right)=0$. Now set $V\left(2^{n+1}\right):=K m_{1}+K m_{2}$ and $U\left(2^{n+1}\right):=U\left(2^{n}\right) V\left(2^{n}\right)+V\left(2^{n}\right) U\left(2^{n}\right)+U\left(2^{n}\right) U\left(2^{n}\right)+$ $P$. Certainly, conditions 1, 3, 5, 6, 7 hold (and 2 does not apply to this case), and condition 5 holds by the observation from the beginning of the proof of this theorem. We claim that condition 4 holds. Indeed, $\bar{f}_{j} \in P \subseteq U\left(2^{n+1}\right)$ and $g_{j} \subseteq U\left(2^{n+1}\right)$ 
for every $1 \leq j \leq s$, so each $f_{j} \in U\left(2^{n+1}\right)$. Therefore $F_{i} \subseteq U\left(2^{n+1}\right)=U\left(2^{2^{i}}\right)$, as required.

Finally, to finish Case 3, consider the case that $i \notin Z$. In this case, take any two monomials $q_{1}, q_{2}$ from $V\left(2^{n}\right) V\left(2^{n}\right)$ and set $V\left(2^{n+1}\right):=K q_{1}+K q_{2}$. Let $Q$ be a $K$-linear subspace of $V\left(2^{n}\right) V\left(2^{n}\right)$ such that $K q_{1}+K q_{2}+Q=V\left(2^{n}\right) V\left(2^{n}\right)$ and $Q \cap\left(K q_{1}+K q_{2}\right)=0$. Now set $V\left(2^{n+1}\right)=K q_{1}+K q_{2}$ and $U\left(2^{n+1}\right)=$ $U\left(2^{n}\right) V\left(2^{n}\right)+V\left(2^{n}\right) U\left(2^{n}\right)+U\left(2^{n}\right) U\left(2^{n}\right)+Q$. It is easy to check all conditions, as in previous cases, noting that $2^{n+1}=2^{2^{i}}$ but $i \notin Z$, so that condition 4 holds trivially.

\section{THE IDEAL $E$}

The algebra we require will be presented as a factor algebra $\bar{A} / E$ for an ideal $E$ that we now define.

Definition 4. Let $r \in H(n)$ for some $n$, and let $m$ be the natural number such that $2^{m} \leq n<2^{m+1}$. We say that $r \in E(n)$ if and only if for all $0 \leq j \leq 2^{m+2}-n$ we have

$$
H(j) r H\left(2^{m+2}-j-n\right) \subseteq U\left(2^{m+1}\right) H\left(2^{m+1}\right)+H\left(2^{m+1}\right) U\left(2^{m+1}\right) .
$$

We define $E=E(1)+E(2)+\ldots$.

Of course, it is not obvious from this definition that $E$ is an ideal of $\bar{A}$. This is the content of the next theorem.

Theorem 5. The set $E$ is a two-sided ideal of $\bar{A}$.

Proof. It suffices to show that if $r \in E(n)$ for some $n$, then $r H(1) \subseteq E(n+1)$ and $H(1) r \subseteq E(n+1)$. Let $m$ be the natural number such that $2^{m} \leq n<2^{m+1}$.

Consider first the case where $n<2^{m+1}-1$. Since $r \in E_{n}$ we know that

$$
H(j) r H\left(2^{m+2}-j-n\right) \subseteq U\left(2^{m+1}\right) H\left(2^{m+1}\right)+H\left(2^{m+1}\right) U\left(2^{m+1}\right)
$$

for all $j \leq 2^{m+2}-n$ and this implies that

$$
H(j) r H(1) H\left(2^{m+2}-j-n-1\right) \subseteq U\left(2^{m+1}\right) H\left(2^{m+1}\right)+H\left(2^{m+1}\right) U\left(2^{m+1}\right)
$$

and

$$
H(j) H(1) r H\left(2^{m+2}-j-n-1\right) \subseteq U\left(2^{m+1}\right) H\left(2^{m+1}\right)+H\left(2^{m+1}\right) U\left(2^{m+1}\right)
$$

for all $j \leq 2^{m+2}-n-1$. Consequently $r H(1) \subseteq E_{n+1}$ and $H(1) r \subseteq E_{n+1}$.

Next, consider the case where $n=2^{m+1}-1$. We have to show that

$$
H(j) H(1) r H\left(2^{m+3}-j-n-1\right) \subseteq U\left(2^{m+2}\right) H\left(2^{m+2}\right)+H\left(2^{m+2}\right) U\left(2^{m+2}\right)
$$

and that

$$
H(j) r H(1) H\left(2^{m+3}-j-n-1\right) \subseteq U\left(2^{m+2}\right) H\left(2^{m+2}\right)+H\left(2^{m+2}\right) U\left(2^{m+2}\right)
$$

for all $j \leq 2^{m+3}-n-1$. Consequently we have to show that

$$
H(j) r H\left(2^{m+3}-n-j\right) \subseteq U\left(2^{m+2}\right) H\left(2^{m+2}\right)+H\left(2^{m+2}\right) U\left(2^{m+2}\right)
$$

for all $j \leq 2^{m+3}-n$.

There are three possibilities to consider:

1. $j+n \leq 2^{m+2}$.

2. $j \geq 2^{m+2}$.

3. $2^{m+2}<j+n<2^{m+2}+n$. 
Case 1. Suppose that $j+n \leq 2^{m+2}$. Since $r \in E(n)$ we have

$H(j) r H\left(2^{m+2}-n-j\right) \subseteq U\left(2^{m+1}\right) H\left(2^{m+1}\right)+H\left(2^{m+1}\right) U\left(2^{m+1}\right) \subseteq U\left(2^{m+2}\right)$,

by Theorem $[3$, condition 6 . Consequently

$$
H(j) r H\left(2^{m+3}-n-j\right) \subseteq U\left(2^{m+2}\right) H\left(2^{m+2}\right),
$$

as required.

Case 2. Suppose that $j \geq 2^{m+2}$. Since $r \in E(n)$ we have $H\left(j-2^{m+2}\right) r H\left(2^{m+2}-n-j\right) \subseteq U\left(2^{m+1}\right) H\left(2^{m+1}\right)+H\left(2^{m+1}\right) U\left(2^{m+1}\right) \subseteq U\left(2^{m+2}\right)$, by Theorem 3 , condition 6 . Consequently

$$
H(j) r H\left(2^{m+3}-n-j\right) \subseteq H\left(2^{m+2}\right) U\left(2^{m+2}\right),
$$

as required.

Case 3. Suppose that $2^{m+2}<j+n<2^{m+2}+n$. Since $n=2^{m+1}-1$, we obtain $2^{m+1}+1<j$ and $2^{m+3}-j-n>2^{m+1}$. Observe that

$$
H(j) r H\left(2^{m+3}-j-n\right) \subseteq H\left(2^{m+1}\right) H(t) r H\left(t^{\prime}\right) H\left(2^{m+1}\right)
$$

for some $t, t^{\prime}$, where $t+t^{\prime}=2^{m+2}-n$ (recall that $n=\operatorname{deg} r$ ).

Since $r \in E(n)$, we obtain

$$
H(t) r H\left(t^{\prime}\right) \subseteq U\left(2^{m+1}\right) H\left(2^{m+1}\right)+H\left(2^{m+1}\right) U\left(2^{m+1}\right) .
$$

Consequently,

$$
\begin{aligned}
H(j) & r H\left(2^{m+3}-j-n\right) \\
& =H\left(2^{m+1}\right) H(t) r H\left(t^{\prime}\right) H\left(2^{m+1}\right) \\
& =H\left(2^{m+1}\right)\left[U\left(2^{m+1}\right) H\left(2^{m+1}\right)+H\left(2^{m+1}\right) U\left(2^{m+1}\right)\right] H\left(2^{m+1}\right) \\
& \subseteq U\left(2^{m+2}\right) H\left(2^{m+2}\right)+H\left(2^{m+2}\right) U\left(2^{m+2}\right),
\end{aligned}
$$

because $H\left(2^{m+1}\right) U\left(2^{m+1}\right) \subseteq U\left(2^{m+2}\right)$ and $U\left(2^{m+1}\right) H\left(2^{m+1}\right) \subseteq U\left(2^{m+2}\right)$, by Theorem 3 , condition 6 .

\section{Definition of $S(j), W(j), R(j), Q(j)$}

In Section 2, the sets $U(*)$ and $V(*)$ were only defined at powers of 2 . In this section we define corresponding sets at all other natural numbers $j$. These are defined in terms of the $U\left(2^{n}\right)$ and $V\left(2^{n}\right)$ for terms occuring in the binary expansion of $j$.

Let $j$ be a natural number. Write $j$ in binary form as

$$
j=2^{p_{0}}+2^{p_{1}}+\ldots+2^{p_{n}}
$$

with $0 \leq p_{0}<p_{1}<\ldots<p_{n}$.

Define

$$
W(j):=V\left(2^{p_{0}}\right) V\left(2^{p_{1}}\right) \ldots V\left(2^{p_{n}}\right)=\prod_{i=0}^{n} V\left(2^{p_{i}}\right)
$$

and set

$$
S(j):=\sum_{k=0}^{n} S(j, k)
$$

with

$$
S(j, 0):=U\left(2^{p_{0}}\right) H\left(j-2^{p_{0}}\right) \quad \text { and } \quad S(j, k):=H\left(t_{k}\right) U\left(2^{p_{k}}\right) H\left(m_{k}\right)
$$


where

for each $j, k>0$.

$$
t_{k}=\sum_{i=0}^{k-1} 2^{p_{i}} \quad \text { and } \quad m_{k}=\sum_{i=k+1}^{n} 2^{p_{i}}
$$

In a similar way, define

$$
Q(j):=V\left(2^{p_{n}}\right) V\left(2^{p_{n-1}}\right) \ldots V\left(2^{p_{0}}\right)=\prod_{i=0}^{n} V\left(2^{p_{n-i}}\right)
$$

and set

$$
R(j):=\sum_{k=0}^{n} R(j, k)
$$

with

$$
R(j, 0):=H\left(j-2^{p_{0}}\right) U\left(2^{p_{0}}\right) \quad \text { and } \quad R(j, k)=H\left(m_{k}\right) U\left(2^{p_{k}}\right) H\left(t_{k}\right),
$$

where

$$
t_{k}=\sum_{i=0}^{k-1} 2^{p_{i}} \quad \text { and } \quad m_{k}=\sum_{i=k+1}^{n} 2^{p_{i}}
$$

for each $j, k>0$.

Note that $W\left(2^{n}\right)=Q\left(2^{n}\right)=V\left(2^{n}\right)$ and that $S\left(2^{n}\right)=R\left(2^{n}\right)=U\left(2^{n}\right)$.

Lemma 6. Let $j$ be a natural number. Then

1. $S(j)+W(j)=H(j)$ and $S(j) \cap W(j)=0$,

2. $R(j)+Q(j)=H(j)$ and $R(j) \cap Q(j)=0$.

Proof. Note that $S(j) \subseteq H(j)$ and $W(j) \subseteq H(j)$ for all $j$. Since $V\left(2^{p_{i}}\right)+U\left(2^{p_{i}}\right)=$ $H\left(2^{p_{i}}\right)$ for all $i$ by Theorem 3 , condition 5 , we get $S(j)+W(j)=H(j)$. Observe that $V\left(2^{p_{i}}\right) \cap U\left(2^{p_{i}}\right)=0$ for all $i$ by Theorem 3 , condition 5 . Therefore $S(j) \cap W(j)=0$.

The proof of the second claim is similar.

Lemma 7. Let $j$ be a natural number, and let $j=2^{p_{0}}+2^{p_{1}}+\ldots+2^{p_{n}}$ be the binary form of $j$ with $0 \leq p_{0}<p_{1}<p_{2}<\ldots<p_{n}$. Let $0<t<n$ and let $m=2^{p_{t}}+2^{p_{t+1}}+\ldots+2^{p_{n}}$ and $m^{\prime}=2^{p_{0}}+2^{p_{1}}+\ldots+2^{p_{t-1}}$. Then $R(j)=$ $R(m) H\left(m^{\prime}\right)+H(m) R\left(m^{\prime}\right)$.

Proof. Notice that $m^{\prime}+m=j$. Let $R(j)=\sum_{i=0}^{n} R(j, k)$ be as in the definition above. Then

where

$$
R(j, k)=H\left(m_{k}\right) U\left(2^{p_{k}}\right) H\left(l_{k}\right)
$$

$$
l_{k}=\sum_{i=0}^{k-1} 2^{p_{i}} \text { and } m_{k}=\sum_{i=k+1}^{n} 2^{p_{i}} .
$$

Suppose that $k<t$, so that $m_{k} \geq m$. Then

$$
R(j, k)=H(m) H\left(m_{k}-m\right) U\left(2^{p_{k}}\right) H\left(l_{k}\right) .
$$

Observe now that $m^{\prime}=\sum_{i=0}^{t-1} 2^{p_{i}}$ is the binary form of $m^{\prime}=j-m$. Therefore $R\left(m^{\prime}, k\right)=H\left(m_{k}-m\right) U\left(2^{p_{k}}\right) H\left(l_{k}\right)$ for $k<t$. Hence $R(j, k)=H(m) R\left(m^{\prime}, k\right)$ for $k<t$, and consequently

$$
\sum_{i=0}^{t-1} R(j, k)=H(m) R\left(m^{\prime}\right)
$$


Now suppose that $k \geq t$, so that $l_{k} \geq m^{\prime}$. Then

$$
R(j, k)=H\left(m_{k}\right) U\left(2^{p_{k}}\right) H\left(l_{k}-m^{\prime}\right) H\left(m^{\prime}\right),
$$

and, arguing as above, $R(j, k)=R(m, k-t) H\left(m^{\prime}\right)$. Therefore,

$$
\sum_{i=t}^{n} R(j, k)=R(m) H\left(m^{\prime}\right)
$$

The result follows.

Theorem 8. For all natural numbers $j, t$ we have

$$
R(j) H(t) \subseteq R(j+t) \quad \text { and } \quad H(t) S(j) \subseteq S(j+t) .
$$

Proof. It is sufficient to show that for every $j$ we have $R(j) H(1) \subseteq R(j+1)$ and $H(1) S(j) \subseteq S(j+1)$. We will show that $R(j) H(1) \subseteq R(j+1)$. The proof that $H(1) S(j) \subseteq S(j+1)$ is similar.

First, consider the case where $j=2^{p+1}-1$ for some $p \geq 0$. Then $j=2^{0}+2^{1}+$ $2^{2}+\ldots+2^{p}$. Consequently $R(j)=\sum_{k=0}^{p} R(j, k)$, where

$$
R(j, k)=H\left(2^{p+1}-2^{k+1}\right) U\left(2^{k}\right) H\left(2^{k}-1\right) .
$$

Notice that $R(j+1)=R\left(2^{p+1}\right)=U\left(2^{p+1}\right)$. Therefore, it suffices to show that $R(j, k) H(1) \subseteq R(j+1)=U\left(2^{p+1}\right)$, for every $k \geq 0$. Notice that

$R(j, k) H(1)=H\left(2^{p+1}-2^{k+1}\right) U\left(2^{k}\right) H\left(2^{k}-1\right) H(1)=H\left(2^{p+1}-2^{k+1}\right) U\left(2^{k}\right) H\left(2^{k}\right)$.

Hence,

$$
R(j, k) H(1) \subseteq H\left(2^{p+1}-2^{k+1}\right) U\left(2^{k+1}\right),
$$

by Theorem 3 , condition 6 . Since $H\left(2^{t}\right) U\left(2^{t}\right) \subseteq U\left(2^{t+1}\right)$, again by Theorem 3 , condition 6 , we obtain

$$
H\left(2^{p+1}-2^{t}\right) U\left(2^{t}\right) \subseteq H\left(2^{p+1}-2^{t+1}\right) U\left(2^{t+1}\right) .
$$

Applying this observation several times for $t=k+1, t=k+2, \ldots, t=p$, we get that $R(j, k) H(1) \subseteq U\left(2^{p+1}\right)$, as required.

Next, assume that $j \neq 2^{p+1}-1$ for all $p$. Write $j$ in binary form: $j=2^{p_{0}}+2^{p_{1}}+$ $\ldots+2^{p_{n}}$ for some $0 \leq p_{0}<p_{1}<p_{2}<\ldots<p_{n}$.

First, assume that $p_{0} \neq 0$. Then $j+1=2^{0}+2^{p_{0}}+2^{p_{1}}+\ldots+2^{p_{n}}$ is the binary form of $j+1$. Let $R(j)=\sum_{i=0}^{n} R(j, k)$ and $R(j+1)=\sum_{i=0}^{n+1} R(j+1, k)$ be as in the definition. Now we see that $R(j, k) H(1) \subseteq R(j+1, k+1)$. Therefore $R(j) H(1) \subseteq R(j+1)$, as required.

Next, assume that $p_{0}=0$, and let $t$ be minimal such that $p_{t}-p_{t-1}>1$. Then $p_{i}=i$ for all $0 \leq i \leq t-1$ and $p_{t}>t$. Therefore $j=2^{t}-1+\sum_{i=t}^{n} 2^{p_{i}}$. By using Lemma 7, observe that $R(j)=R(m) H\left(m^{\prime}\right)+H(m) R\left(m^{\prime}\right)$ where $m^{\prime}=\sum_{i=0}^{t-1} 2^{p_{i}}=$ $2^{t}-1$ and $m=\sum_{i=t}^{n} 2^{p_{i}}$. Thus,

$$
R(j) H(1)=R(m) H\left(m^{\prime}\right) H(1)+H(m) R\left(m^{\prime}\right) H(1) .
$$

Since $m^{\prime}=2^{t}-1$, we get $R\left(m^{\prime}\right) H(1) \subseteq R\left(m^{\prime}+1\right)=R\left(2^{t}\right)$, by the first part of the proof. Therefore

$$
R(j) H(1) \subseteq R(m) H\left(m^{\prime}+1\right)+H(m) R\left(m^{\prime}+1\right) .
$$


Observe that the binary form of $j+1$ is $j+1=2^{t}+\sum_{i=t}^{n} 2^{p^{i}}$. Recall that $m=\sum_{i=t}^{n} 2^{p^{i}}$ and that $2^{t}=m^{\prime}+1$. Now from Lemma 7 we get

$$
R(j+1)=R(m) H\left(m^{\prime}+1\right)+H(m) R\left(m^{\prime}+1\right) .
$$

Consequently $R(j) H(1) \subseteq R(j+1)$, and the lemma follows.

\section{Estimation of the Gelfand-Kirillov dimension}

In order to estimate the Gelfand-Kirillov dimension of $\bar{A} / E$, we need to recognise when certain homogenous elements are in $E$. The next theorem provides a sufficient condition for this to happen.

Theorem 9. If $r \in H(n)$ for some $n$ and $r \in S(t) H(n-t)+H(t) R(n-t)$ for all $0 \leq t \leq n$, then $r \in E$.

Proof. Suppose that $r \in S(t) H(n-t)+H(t) R(n-t)$ for all $0 \leq t \leq n$. Let $m$ be the natural number such that $2^{m} \leq n<2^{m+1}$. By the definition of $E$ we have to show that for all $j \leq 2^{m+2}-n$ we have

$$
H(j) r H\left(2^{m+2}-j-n\right) \subseteq U\left(2^{m+1}\right) H\left(2^{m+1}\right)+H\left(2^{m+1}\right) U\left(2^{m+1}\right) .
$$

Consider the three possibilities:

1. $j+n>2^{m+1}$ and $2^{m+1}-j \geq 0$.

2. $j+n>2^{m+1}$ and $2^{m+1}-j<0$.

3. $j+n \leq 2^{m+1}$.

Case 1. Suppose first that $j+n>2^{m+1}$ and $2^{m+1}-j \geq 0$. Set $t=2^{m+1}-j$. By assumption, $r \in S(t) H(n-t)+H(t) R(n-t)$, since $0 \leq t$ and $n-t=n+j-2^{m+1}>0$. Therefore

$H(j) r H\left(2^{m+2}-j-n\right) \subseteq H(j)\{S(t) H(n-t)+H(t) R(n-t)\} H\left(2^{m+2}-j-n\right)$.

Now, since $j+t=2^{m+1}$, we get

$H(j) r H\left(2^{m+2}-j-n\right) \subseteq H(j) S(t) H\left(2^{m+1}\right)+H\left(2^{m+1}\right) R(n-t) H\left(2^{m+1}-n+t\right)$.

By Theorem $8, H(j) S(t) \subseteq S\left(2^{m+1}\right)=U\left(2^{m+1}\right)$. Similarly,

$$
R(n-t) H\left(2^{m+1}-n+t\right) \subseteq R\left(2^{m+1}\right)=U\left(2^{m+1}\right),
$$

by Theorem 8 , Therefore

$$
H(j) r H\left(2^{m+2}-j-n\right) \subseteq U\left(2^{m+1}\right) H\left(2^{m+1}\right)+H\left(2^{m+1}\right) U\left(2^{m+1}\right),
$$

as required.

Case 2. Suppose that $j+n>2^{m+1}$ and $j>2^{m+1}$. Then $j=2^{m+1}+b$ for some $b>0$. Since $j+n \leq 2^{m+2}$, it follows that $b+n \leq 2^{m+1}$; and $b \leq 2^{m}$, since $n \geq 2^{m}$. Now take $t=2^{m}-b$. Then $0 \leq t \leq n$. Hence, by assumption, $r \in S(t) H(n-t)+H(t) R(n-t)$. Consequently,

$H(j) r H\left(2^{m+2}-j-n\right) \subseteq H(j)\{S(t) H(n-t)+H(t) R(n-t)\} H\left(2^{m+2}-j-n\right)$.

Since $j=2^{m+1}+b$ we obtain

$$
\begin{aligned}
H(j) r H\left(2^{m+2}-j-n\right) \subseteq & H\left(2^{m+1}\right) H(b)\{S(t) H(n-t)+H(t) R(n-t)\} \\
& \times H\left(2^{m+2}-j-n\right) .
\end{aligned}
$$

Consider the two terms that occur on the right hand side of this containment separately. 
First, consider the term $H\left(2^{m+1}\right) H(b) S(t) H(n-t) H\left(2^{m+2}-j-n\right)$. Note that $t+b=2^{m}$ and that $j+t=2^{m+1}+2^{m}$. Hence, $H(b) S(t) \subseteq S\left(2^{m}\right)=U\left(2^{m}\right)$, by Theorem 8 and so

$$
\begin{aligned}
H\left(2^{m+1}\right) H(b) S(t) H(n-t) H\left(2^{m+2}-j-n\right) & =H\left(2^{m+1}\right) U\left(2^{m}\right) H\left(2^{m+2}-j-t\right) \\
& =H\left(2^{m+1}\right) U\left(2^{m}\right) H\left(2^{m}\right) \\
& \subseteq H\left(2^{m+1}\right) U\left(2^{m+1}\right),
\end{aligned}
$$

as required.

Next, consider the term $H\left(2^{m+1}\right) H(b) H(t) R(n-t) H\left(2^{m+2}-j-n\right)$.

Observe that $R(n-t) H\left(2^{m+2}-j-n\right) \subseteq R\left(2^{m}\right)=U\left(2^{m}\right)$, by Theorem 8 , since $j+t=2^{m+1}+2^{m}$. Also, $H(b) H(t)=H(t+b)=H\left(2^{m}\right)$.

Hence,

$$
\begin{aligned}
H\left(2^{m+1}\right) H(b) H(t) R(n-t) H\left(2^{m+2}-j-n\right) & \subseteq H\left(2^{m+1}\right) H\left(2^{m}\right) U\left(2^{m}\right) \\
& \subseteq H\left(2^{m+1}\right) U\left(2^{m+1}\right)
\end{aligned}
$$

as required.

Consequently, $H(j) r H\left(2^{m+2}-j-n\right) \subseteq H\left(2^{m+1}\right) U\left(2^{m+1}\right)$, as required.

Case 3. Suppose that $j+n \leq 2^{m+1}$. Then $j \leq 2^{m}$, since $n \geq 2^{m}$. Set $t:=2^{m}-j$. Then $0 \leq t \leq n$. By assumption, $r \in S(t) H(n-t)+H(t) R(n-t)$. Note that $j+t=2^{m}$. Therefore,

$$
H(j) r H\left(2^{m+2}-n-j\right) \subseteq H(j)\{S(t) H(n-t)+H(t) R(n-t)\} H\left(2^{m+2}-n-j\right) .
$$

Theorem 8 gives $H(j) S(t) \subseteq S(j+t)=S\left(2^{m}\right)=U\left(2^{m}\right)$, so that

$H(j) r H\left(2^{m+2}-n-j\right) \subseteq U\left(2^{m}\right) H\left(2^{m+2}-j-t\right)+H\left(2^{m}\right) R(n-t) H\left(2^{m+2}-n-j\right)$.

Consider the two terms on the right hand side of this containment.

First,

$$
\begin{aligned}
U\left(2^{m}\right) H\left(2^{m+2}-j-t\right) & =U\left(2^{m}\right) H\left(2^{m+2}-2^{m}\right)=U\left(2^{m}\right) H\left(2^{m}\right) H\left(2^{m+1}\right) \\
& =U\left(2^{m+1}\right) H\left(2^{m+1}\right) .
\end{aligned}
$$

Second, note that $2^{m}-n+t=2^{m}-n+\left(2^{m}-j\right)=2^{m+1}-(n+j) \geq 0$ and that $2^{m+2}-n-j=2^{m+1}+2^{m+1}-n-j=2^{m+1}+\left(2^{m}-n+t\right)$, and so

$$
\begin{aligned}
H\left(2^{m}\right) R(n-t) H\left(2^{m+2}-n-j\right) & =H\left(2^{m}\right) R(n-t) H\left(2^{m}-n+t\right) H\left(2^{m+1}\right) \\
& \subseteq H\left(2^{m}\right) R\left(2^{m}\right) H\left(2^{m+1}\right) \\
& =H\left(2^{m}\right) U\left(2^{m}\right) H\left(2^{m+1}\right) \subseteq U\left(2^{m+1}\right) H\left(2^{m+1}\right),
\end{aligned}
$$

as required.

Consequently $H(j) r H\left(2^{m+2}-n-j\right) \subseteq U\left(2^{m+1}\right) H\left(2^{m+1}\right)$. This finishes the proof.

We can now estimate the size of the subspaces $Q(n)$ and $W(n)$.

Theorem 10. For all $n>0$, we have $\operatorname{dim}_{K} Q(n) \leq 3^{17} n^{9}$ and $\operatorname{dim}_{K} W(n) \leq$ $3^{17} n^{9}$.

Proof. We will show that $\operatorname{dim}_{K} Q(n) \leq 3^{17} n^{9}$. The proof that $\operatorname{dim}_{K} W(n) \leq 3^{17} n^{9}$ is similar. Let $n=2^{p_{0}}+2^{p_{1}}+\ldots+2^{p_{m}}$ with $0 \leq p_{0}<p_{1}<p_{2}<\ldots<p_{m}$. Then $Q(n)=V\left(2^{p_{0}}\right) V\left(2^{p_{1}}\right) \ldots V\left(2^{p_{m}}\right)$. Consequently $\operatorname{dim}_{K} Q(n)=\prod_{i=0}^{m} \operatorname{dim}_{K} V\left(2^{p_{i}}\right)$. Therefore $\operatorname{dim}_{K} Q(n) \leq \prod_{i=0}^{\lfloor\log (n)\rfloor} \operatorname{dim}_{K} V\left(2^{i}\right)$, where $\lfloor\log (n)\rfloor$ is the largest integer 
not exceeding $\log (n)$. Recall that, from Theorem 2, either $\operatorname{dim}_{K} V\left(2^{i}\right)=2$ or $i \in S$, where $S=\left\{\left[2^{i}-i-1,2^{i}-1\right] \mid i=5,6, \ldots\right\}$.

Now let $c_{i}=\prod_{t=2^{i}-i-1}^{2^{i}-1} \operatorname{dim}_{K} V\left(2^{t}\right)$. We see that $c_{i}=\prod_{j=0}^{i} \operatorname{dim}_{K} V\left(2^{2^{i}-i-1+j}\right)$ $=\prod_{j=0}^{i} 2^{2^{j}}<2^{2^{i+1}}$, by Theorem 3, condition 2. Since

$$
\operatorname{dim}_{K} Q(n) \leq \prod_{i=0}^{\lfloor\log (n)\rfloor} \operatorname{dim}_{K} V\left(2^{i}\right),
$$

we have $\operatorname{dim}_{K} Q(n) \leq c c^{\prime}$ where

$$
c=\prod_{i \notin S, i \leq\lfloor\log (n)\rfloor} \operatorname{dim}_{K} V\left(2^{i}\right)
$$

and

$$
c^{\prime}=\prod_{i \in S, i \leq\lfloor\log (n)\rfloor} \operatorname{dim}_{K} V\left(2^{i}\right) .
$$

First, observe that $c \leq 2^{\lfloor\log (n)\rfloor+1} \leq 2 n$. Next, let $q$ be the maximal number such that $2^{q}-q-1 \leq\lfloor\log (n)\rfloor$. Then $c^{\prime} \leq \prod_{i=0}^{q} c_{i} \leq \prod_{i=0}^{q} 2^{2^{q+1}} \leq 2^{2^{q+2}}$. Observe that $2^{q-1} \leq 2^{q}-(q+1)$, if $q \geq 3$. Therefore $2^{2^{q+2}}=2^{2^{q-1} 2^{3}}=\left(2^{2^{q-1}}\right)^{8} \leq$ $\left(2^{\lfloor\log (n)\rfloor}\right)^{8} \leq n^{8}$, provided that $q \geq 3$. Observe that if $n \geq 16$, then the maximal number $q$ with $2^{q}-q-1 \leq\lfloor\log (n)\rfloor$ is indeed greater than or equal to 3 , so that $\operatorname{dim}_{K}(Q(n)) \leq 2 n . n^{8}=2 n^{9}$ provided that $n \geq 16$. If $n<16$, then $\operatorname{dim}_{K}(Q(n)) \leq$ $\operatorname{dim}_{K}(H(n))<3^{16}$, since $Q(n) \subseteq H(n)$ for each $n$ and $A$ is generated by the three elements $x, y, z$. Therefore $\operatorname{dim}_{k} Q(n) \leq 3^{17} n^{9}$ for each $n$.

After all this preparation, we can now estimate the Gelfand-Kirillov dimension of our factor algebra.

Theorem 11. GKdim $(\bar{A} / E) \leq 20$.

Proof. Let $0 \leq j \leq n$. Note that $S(j)+W(j)=H(j)$ and $R(n-j)+Q(n-j)=$ $H(n-j)$. It follows that

$$
H(n)=H(j) H(n-j)=W(j) Q(n-j)+\{S(j) H(n-j)+H(j) R(n-j)\} .
$$

Thus,

$\operatorname{dim}\left(\frac{H(n)}{\{S(j) H(n-j)+H(j) R(n-j)\}}\right) \leq \operatorname{dim} W(j) Q(n-j) \leq\left(3^{17} n^{9}\right)^{2}=3^{34} n^{18}$.

Let

$$
\theta: H(n) \longrightarrow \bigoplus_{j=0}^{n} \frac{H(n)}{\{S(j) H(n-j)+H(j) R(n-j)\}}
$$

be the natural map. Then

$\operatorname{ker}(\theta)=\{r \in H(n) \mid r \in S(j) H(n-j)+H(j) R(n-j)$ for each $0 \leq j \leq n\} \subseteq E(n)$ by Theorem 9 , Thus,

$$
\operatorname{dim}\left(\frac{H(n)}{E(n)}\right) \leq \operatorname{dim}\left(\frac{H(n)}{\operatorname{ker}(\theta)}\right) \leq 3^{34} n^{18}(n+1) .
$$

Consequently, $\operatorname{GKdim}(\bar{A} / E) \leq 20$. 


\section{6. $\bar{A} / E$ IS NIL BUt NOT Nilpotent}

It remains to show that the algebra $\bar{A} / E$ is nil but not nilpotent. We show that $\bar{A} / E$ is nil by showing that the elements $f_{i}{ }^{10 w_{i}}$ defined in Section 1 belong to $E$. In order to see that $\bar{A} / E$ is not nilpotent we show that the $K$-subspaces $V\left(2^{n}\right)$ are not contained in $E$.

Lemma 12. Let $Z,\left\{f_{i}\right\}_{i \in Z},\left\{F_{i}\right\}_{i \in Z}$ be as in Theorem 2. Fix any $i \in Z$ and suppose that $m+2>2^{i}$. Then

$$
B_{w_{i}}\left(F_{i}\right) \cap H\left(2^{m+2}\right) \subseteq U\left(2^{m+1}\right) H\left(2^{m+1}\right)+H\left(2^{m+1}\right) U\left(2^{m+1}\right) .
$$

Proof. We know that $F_{i} \subseteq H\left(2^{2^{i}}\right)$ and $w_{i}=4 r_{i}$ where $r_{i}=2^{2^{i}}$. Set $w:=w_{i}$ and $r:=r_{i}$. By the assumptions of this lemma $2^{m+1} \geq 2^{2^{i}}$. Observe that $B_{w}\left(F_{i}\right) \subseteq$ $B_{r}\left(F_{i}\right)$, since $w=4 r$. Also, $B_{r}\left(F_{i}\right) \subseteq B_{r}(U(r))$, since $F_{i} \subseteq U\left(2^{2^{i}}\right)=U(r)$ by Theorem 3 condition 4 . Consequently

$$
B_{w}\left(F_{i}\right) \subseteq B_{r}(U(r)) .
$$

Therefore, it is sufficient to show that

$$
B_{r}\left(U_{r}\right) \cap H\left(2^{m+2}\right) \subseteq U\left(2^{m+1}\right) H\left(2^{m+1}\right)+H\left(2^{m+1}\right) U\left(2^{m+1}\right)
$$

for all $m$ such that $2^{m+1} \geq 2^{2^{i}}=r$. We will proceed by induction on $m$. If $m+1=2^{i}$, then $2^{m+2}=2 r$, so that $B_{r}(U(r)) \cap H(2 r)=U(r) H(r)+H(r) U(r)$, by the definition of $B_{r}(U(r))$, and the fact that $U(r) \subseteq H(r)$.

Suppose now that the result holds for some $m$, with $2^{m+1} \geq 2^{2^{i}}=r$. We will prove that the result holds for $m+1$. We have to show that

$$
B_{r}(U(r)) \cap H\left(2^{m+3}\right) \subseteq U\left(2^{m+2}\right) H\left(2^{m+2}\right)+H\left(2^{m+2}\right) U\left(2^{m+2}\right) .
$$

Observe that, since $r$ divides $2^{m+2}$, we obtain

$$
\begin{aligned}
B_{r}(U(r)) \cap H\left(2^{m+3}\right)= & \left\{B_{r}(U(r)) \cap H\left(2^{m+2}\right)\right\} H\left(2^{m+2}\right) \\
& +H\left(2^{m+2}\right)\left\{B_{r}(U(r)) \cap H\left(2^{m+2}\right)\right\},
\end{aligned}
$$

by the definition of $B_{r}(U(r))$. By the induction assumption

$$
B_{r}(U(r)) \cap H\left(2^{m+2}\right) \subseteq U\left(2^{m+1}\right) H\left(2^{m+1}\right)+H\left(2^{m+1}\right) U\left(2^{m+1}\right) \subseteq U\left(2^{m+2}\right)
$$

by Theorem 3 , condition 6 . Hence,

$$
B_{r}(U(r)) \cap H\left(2^{m+3}\right) \subseteq U\left(2^{m+2}\right) H\left(2^{m+2}\right)+H\left(2^{m+2}\right) U\left(2^{m+2}\right)
$$

and the result follows.

Theorem 13. Let $Z,\left\{f_{i}\right\}_{i \in Z}$ be as in Lemma 1. Let $i \in Z$ and let $I$ be the two sided ideal of $R$ generated by $f_{i}^{10 w_{i}}$ where $w_{i}=4.2^{2^{i}}$. Then $I \subseteq E$.

Proof. Let $r \in I$. Then $r=\sum_{p=10 w_{i}}^{s} r_{p}$ for some $r_{p} \in H(p)$, and some $s$. Fix $n$, with $10 w_{i} \leq n \leq s$. It is sufficient to show that $r_{n} \in E$. Let $m$ be the natural number such that $2^{m} \leq n<2^{m+1}$. Note that $10 w_{i}=40.2^{2^{i}}$, so $40.2^{2^{i}} \leq n<2^{m+1}$. Hence $m+1>2^{i}$. In order to show that $r_{n} \in E$, we have to show that

$$
H(j) r_{n} H\left(2^{m+2}-n-j\right) \subseteq U\left(2^{m+1}\right) H\left(2^{m+1}\right)+H\left(2^{m+1}\right) U\left(2^{m+1}\right),
$$

for every $0 \leq j \leq 2^{m+2}-n$.

Now $r \in I$ yields $H(j) r H\left(2^{m+2}-n-j\right) \subseteq I$. Consequently,

$$
H(j) r H\left(2^{m+2}-n-j\right) \subseteq B_{w_{i}}\left(F_{i}\right),
$$


by Theorem 2 , so

$$
H(j)\left(\sum_{p=10 w_{i}}^{s} r_{p}\right) H\left(2^{m+2}-n-j\right) \subseteq B_{w_{i}}\left(F_{i}\right) .
$$

It follows that $H(j) r_{p} H\left(2^{m+2}-n-j\right) \subseteq B_{w_{i}}\left(F_{i}\right)$, for every $p$ with $10 w_{i} \leq p \leq s$, since $B_{w_{i}}\left(F_{i}\right)$ is homogeneous and $r_{p} \in H(p)$ for every $p$.

In particular,

$$
H(j) r_{n} H\left(2^{m+2}-n-j\right) \subseteq B_{w_{i}}\left(F_{i}\right) .
$$

Now, since $H(j) r_{n} H\left(2^{m+2}-n-j\right) \subseteq H\left(2^{m+2}\right)$ and $m+2>2^{i}$, we have

$$
\begin{aligned}
H(j) r_{n} H\left(2^{m+2}-n-j\right) & \subseteq B_{w_{i}}\left(F_{i}\right) \cap H\left(2^{m+2}\right) \\
& \subseteq H\left(2^{m+1}\right) U\left(2^{m+1}\right)+U\left(2^{m+1}\right) H\left(2^{m+1}\right),
\end{aligned}
$$

by Lemma 12, and this completes the proof.

The next two results are now immediate.

Corollary 14. Let $Z,\left\{f_{i}\right\}_{i \in Z}$ be as in Lemma 1. Let $N$ be the two-sided ideal in A generated by elements from the set $\left\{f_{i}^{10 w_{i}}\right\}_{i \in Z}$ where $w_{i}=4.2^{2^{i}}$. Then $N \subseteq E$.

Theorem 15. The algebra $\bar{A} / E$ is a nil algebra.

Proof. This follows from the previous theorem and Lemma 1.

Finally, we show that $\bar{A} / E$ is not nilpotent.

Theorem 16. The algebra $\bar{A} / E$ is not nilpotent.

Proof. Recall that $V\left(2^{n+1}\right) \subseteq V\left(2^{n}\right) V\left(2^{n}\right)$, for every $n>0$, by Theorem 3 , condition 7. It follows easily, by induction, that $V\left(2^{m}\right) \subseteq V(2)^{2^{m-1}}$. Thus, it is sufficient to show that $V\left(2^{m}\right) \nsubseteq E$.

Recall that, by Theorem 3 , condition 3, $V\left(2^{m}\right)$ is generated by monomials, for all $m$. Therefore, there are $0 \neq r \in V\left(2^{m}\right)$ and $0 \neq r^{\prime} \in H\left(2^{m+2}-2^{m}\right)$ such that $0 \neq r r^{\prime} \in V\left(2^{m+2}\right)$. Suppose that $r \in E$; so that, in fact, $r \in E\left(2^{m}\right)$. By using the defining property of $E$, see Definition 4, with $j=0$ and $n=2^{m}$, we obtain

$$
\begin{aligned}
0 \neq r r^{\prime} \in H(0) r H\left(2^{m+2}-0-2^{m}\right) & \subseteq U\left(2^{m+1}\right) H\left(2^{m+1}\right)+H\left(2^{m+1}\right) U\left(2^{m+1}\right) \\
& \subseteq U\left(2^{m+2}\right) .
\end{aligned}
$$

Thus, $0 \neq r r^{\prime} \in V\left(2^{m+2}\right) \cap U\left(2^{m+2}\right)=0$, contradicting Theorem 3 , condition 5 .

Hence, $r \notin E$, so that $V\left(2^{m}\right) \nsubseteq E$, as required.

In conclusion, we have proved:

Theorem 17. The finitely generated algebra $\bar{A} / E$ is nil, but not nilpotent, and has Gelfand-Kirillov dimension not exceeding 20.

\section{ConCluding REMARKS}

It is clear that with more careful analysis of inequalities the bound on the Gelfand-Kirillov dimension obtained above can be reduced. However, we cannot see that the method we employ here could be used to construct a nil but not locally nilpotent example with Gelfand-Kirillov dimension less than four. In contrast, nil does imply nilpotent for Gelfand-Kirillov dimension one, by [5]. Combining this 
with Bergman's Gap Theorem, 3, Theorem 2.5], we see that a nil but not nilpotent example must have Gelfand-Kirillov dimension at least two. It would be very interesting to find the precise dividing line in terms of growth. A starting point might be to consider nil algebras with quadratic growth.

The methods employed here depend crucially on the countability hypothesis. It would be interesting to see if it is possible to construct an affine infinite dimensional nil algebra with finite Gelfand-Kirillov dimension over an uncountable field.

\section{REFERENCES}

[1] V A Ufnarovskij, Combinatorial and asymptotic methods in algebra, Algebra, VI, 1-196, Encyclopaedia Math. Sci., 57, Springer, Berlin, 1995. MR1360005

[2] E S Golod, and I R Shafarevich, On the class field tower, Izv. Akad. Nauk. SSSR Mat. Ser. 28 (1964), 261-272 (in Russian). MR0161852 (28:5056)

[3] G R Krause and T H Lenagan, Growth of algebras and Gelfand-Kirillov dimension, Revised edition. Graduate Studies in Mathematics, 22. American Mathematical Society, Providence, RI, 2000. MR.1721834 (2000j:16035)

[4] L W Small, private communication, February 2004.

[5] L W Small, J T Stafford and R B Warfield, Jr, Affine algebras of Gelfand-Kirillov dimension one are PI, Math. Proc. Cambridge Philos. Soc. 97 (1985), no. 3, 407-414. MR778674 (86g:16025)

[6] Agata Smoktunowicz, Polynomial rings over nil rings need not be nil, Journal of Algebra 233 (2000), 427-436. MR1793911(2001i:16045)

Maxwell Institute for Mathematical Sciences, School of Mathematics, University of Edinburgh, James Clerk Maxwell Building, King's Buildings, Mayfield Road, EdinBURGH EH9 3JZ, SCOTLAND

E-mail address: tom@maths.ed.ac.uk

Institute of Mathematics, Polish Academy of Sciences, Sniadeckich 8, 00-956 Warsaw 10, Poland

E-mail address: agatasm@impan.gov.pl

Current address: Maxwell Institute for Mathematical Sciences, School of Mathematics, University of Edinburgh, James Clerk Maxwell Building, King's Buildings, Mayfield Road, Edinburgh EH9 3JZ, Scotland 